\title{
Trace formulas for stochastic evolution operators: smooth conjugation method
}

\author{
Predrag Cvitanović†, C P Dettmann†, Ronnie Mainierił \\ and Gábor Vattay§ \\ † Center for Chaos and Turbulence Studies, Niels Bohr Institute \\ Blegdamsvej 17, DK-2100 Copenhagen $\varnothing$ \\ $\ddagger$ Theoretical Division, MS B213 \\ Los Alamos National Laboratory, Los Alamos, NM 87545 \\ $\S$ Dept. Solid State Physics \\ Eötvös University, Muzeum krt. 6-8, H-1088 Budapest
}

\begin{abstract}
The trace formula for the evolution operator associated with nonlinear stochastic flows with weak additive noise is cast in the path integral formalism. We integrate over the neighborhood of a given saddlepoint exactly by means of a smooth conjugacy, a locally analytic nonlinear change of field variables. The perturbative corrections are transfered to the corresponding Jacobian, which we expand in terms of the conjugating function, rather than the action used in defining the path integral. The new perturbative expansion which follows by a recursive evaluation of derivatives appears more compact than the standard Feynman diagram perturbation theory. The result is a stochastic analog of the Gutzwiller trace formula with the " $\hbar$ " corrections computed an order higher than what has so far been attainable in stochastic and quantum-mechanical applications.

PACS numbers: 02.50.Ey, 03.20.+i, 03.65.Sq, 05.40.+j, 05.45.+b
\end{abstract}

AMS classification scheme numbers: $58 \mathrm{~F} 20$

keywords: noise, stochastic dynamics, Fokker-Planck operator, cycle expansions, periodic orbits, semiclassical expansions, perturbative expansions, trace formulas, spectral determinants, zeta functions.

\section{Introduction}

The study of stochastic perturbations of dynamical systems is important in applications to realistic systems since their description contains uncertainties due to degrees of freedom that are not included, either because they are not measurable, or because their inclusion would complicate the analysis. A small stochastic perturbation can smooth the singular distributions inherent in many nonlinear dynamical systems. This makes it easier to study averages over such distributions as well defined 
deterministic limits of smooth stochastic distributions. Besides being an ubiquitous fact of life - any dynamics in nature is stochastic to some degree, however weak stochastic processes offer us a great freedom in picking problems and testing ideas.

The properties of the weakly stochastic system are here determined from the unstable periodic orbits of the unperturbed (deterministic) system, decorated by the stochastic corrections. The central object in the theory, the trace of the evolution operator, is a discrete path integral, similar to those found in field theory and statistical mechanics. The weak noise perturbation theory, likewise, resembles perturbative field theory, and in the preceding paper [1] we developed such a perturbation theory for trace formulas for weakly stochastic chaotic dynamics in the standard field-theoretic language of Feynman diagrams.

Here we approach the same problem from an althogether different direction; the key idea of flattening the neighborhood of a saddlepoint can be traced back to Poincaré [2], and is perhaps not something that a field theorist would instinctively hark to as a method of computing perturbative corrections. In the Feynman diagram approach [i] we observed that the sums of diagrams simplify for saddlepoints corresponding to repeats of shorter periodic orbits, and were surprised by the compactness of the order $\sigma^{2}$ correction. Here we explain this simplification in geometric terms that might be applicable to more general field theoretic problems.

\section{Stochastic evolution operator}

The periodic orbit theory allows us to calculate long time averages in a chaotic system as expansions in terms of the periodic orbits (cycles) of the system. The simplest example is provided by the Perron-Frobenius operator

$$
\mathcal{L} \rho\left(x^{\prime}\right)=\int d x \delta\left(f(x)-x^{\prime}\right) \rho(x)
$$

for a deterministic map $f(x)$ which maps a density distribution $\rho(x)$ forward in time. The periodic orbit theory relates the spectrum of this operator and its weighted evolution operator generalizations to the periodic orbits via trace formulas, dynamical zeta functions and spectral determinants 3 , 田. For quantum mechanics the periodic orbit theory is exact on the semiclassical level [5], whereas the quintessentially quantum effects such as creeping, tunneling and diffraction have to be included as corrections. In particular, the higher order $\hbar$ corrections can be computed perturbatively by means of Feynman diagrammatic expansions [6. Our purpose here is to develop the parallel theory for stochastic dynamics. In case at hand, already a discrete time 1-dimensional discrete Langevin equation [7, 8,

$$
x_{n+1}=f\left(x_{n}\right)+\sigma \xi_{n},
$$

with $\xi_{n}$ independent normalized random variables, suffices to reveal the structure of the perturbative corrections.

We treat a chaotic system with weak external noise by replacing the deterministic evoluton $\delta$-function kernel by $\mathcal{L}_{\sigma}$, the Fokker-Planck kernel corresponding to (1), a sharply peaked noise distribution function

$$
\mathcal{L}_{\sigma}\left(x^{\prime}, x\right)=\delta_{\sigma}\left(f(x)-x^{\prime}\right) .
$$

In ref. [1] we have treated the problem of computing the spectrum of this operator by standard field-theoretic Feynman diagram expansions. This time we formulate 
the perturbative expansion in terms of smooth conjugacies and recursively evaluated derivatives. The procedure, which is relatively easy to automatize, enables us to go one order further in the perturbation theory, with much less computational effort than Feynman diagrammatic expansions would require.

In the weak noise limit the kernel is sharply peaked, so it makes sense to expand it in terms of the Dirac delta function and its derivatives:

$$
\delta_{\sigma}(y)=\sum_{m=0}^{\infty} \frac{a_{m} \sigma^{m}}{m !} \delta^{(m)}(y)=\delta(y)+a_{2} \frac{\sigma^{2}}{2} \delta^{(2)}(y)+a_{3} \frac{\sigma^{3}}{6} \delta^{(3)}(y)+\ldots
$$

where

$$
\delta^{(k)}(y)=\frac{\partial^{k}}{\partial y^{k}} \delta(y),
$$

and the coefficients $a_{m}$ depend on the choice of the kernel. We have omitted the $\delta^{(1)}(y)$ term in the above because in our applications we shall impose the saddle-point condition, that is, we shift $f$ by a constant to ensure that the noise peak corresponds

to $y=0$, so $\delta_{\sigma}^{\prime}(0)=0$. For example, if $\delta_{\sigma}(y)$ is a Gaussian kernel, it can be expanded as

$$
\begin{aligned}
\delta_{\sigma}(y) & =\frac{1}{\sqrt{2 \pi \sigma^{2}}} e^{-y^{2} / 2 \sigma^{2}}=\sum_{n=0}^{\infty} \frac{\sigma^{2 n}}{n ! 2^{n}} \delta^{(2 n)}(y) \\
& =\delta(y)+\frac{\sigma^{2}}{2} \delta^{(2)}(y)+\frac{\sigma^{4}}{8} \delta^{(4)}(y)+\cdots
\end{aligned}
$$

\section{Stochastic trace formula}

We start our computation of the weak noise corrections to the spectrum of $\mathcal{L}_{\sigma}$ by calculating the trace of the $n$th iterate of the stochastic evolution operator $\mathcal{L}_{\sigma}$ for a onedimensional analytic map $f(x)$ with additive noise $\sigma$. This trace is an $n$-dimensional integral on $n$ points along a discrete periodic chain, so $x$ becomes an $n$-vector $x_{a}$ with indices $a, b, \ldots$ ranging from 0 to $n-1$ in a cyclic fashion

$$
\begin{aligned}
\operatorname{tr} \mathcal{L}_{\sigma}^{n} & =\int \prod_{a=0}^{n-1} d x_{a} \delta_{\sigma}\left(y_{a}\right) \\
y_{a}(x) & =f\left(x_{a}\right)-x_{a+1}, \quad x_{n}=x_{0} .
\end{aligned}
$$

To the order $\sigma^{3}$ the composition is simple: all compositions but one can be made, resulting in

$$
\begin{aligned}
\operatorname{tr} \mathcal{L}_{\sigma}^{n}= & \int d x \delta\left(f^{n}(x)-x\right)+\frac{a_{2}}{2} \sigma^{2} \int d x_{1} \ldots d x_{n} \\
& \sum_{a=0}^{n-1} \delta\left(f\left(x_{n}\right)-x_{1}\right) \ldots \delta^{(2)}\left(f\left(x_{a}\right)-x_{a+1}\right) \ldots \delta\left(f\left(x_{1}\right)-x_{2}\right)+\cdots \\
= & \operatorname{tr} \mathcal{L}^{n}+\frac{a_{2}}{2} \sigma^{2} \sum_{a=0}^{n-1} \int d x \delta^{(2)}\left(f^{n}\left(x_{a}\right)-x_{a}\right) \\
& +\frac{a_{3}}{3 !} \sigma^{3} \sum_{a=0}^{n-1} \int d x \delta^{(3)}\left(f^{n}\left(x_{a}\right)-x_{a}\right)+O\left(\sigma^{4}\right) .
\end{aligned}
$$


At fourth order we get contributions from $\delta^{(4)}$, as well as the two-point contributions

$$
\begin{aligned}
\operatorname{tr} \mathcal{L}_{\sigma}^{n}= & (\cdots)+\frac{a_{4}}{4 !} \sigma^{4} \sum_{a=0}^{n-1} \int d x \delta^{(4)}\left(f^{n}\left(x_{a}\right)-x_{a}\right) \\
& +\frac{a_{2}^{2}}{4} \sigma^{4} \sum_{a<b} \int d x_{a} d x_{b} \delta^{(2)}\left(f^{j}\left(x_{a}\right)-x_{b}\right) \delta^{(2)}\left(f^{k}\left(x_{b}\right)-x_{a}\right),(7)
\end{aligned}
$$

where $j$ is the number of steps from points $a$ to $b$ on the cycle, and $k$ is the number of step from $b$ to $a$, so that $j+k=n$.

If the map is smooth, the periodic points of given finite period $n$ are isolated and the noise broadening $\sigma$ sufficiently small so that they remain separated, the dominant contributions come from neighborhoods of periodic points; in the saddlepoint approximation the trace (5) is given by

$$
\operatorname{tr} \mathcal{L}_{\sigma}^{n} \longrightarrow \sum_{x_{c} \in \operatorname{Fix}^{n}} e^{W_{c}},
$$

As traces are cyclic, $e^{W_{c}}$ is the same for all periodic points in a given cycle, independent of the choice of the starting point $x_{c}$. Hence it is customary to rewrite this sum in terms of prime cycles and their repeats,

$$
\left.\operatorname{tr} \mathcal{L}_{\sigma}^{n}\right|_{\text {saddles }}=\sum_{p} n_{p} \sum_{r=1}^{\infty} e^{W_{p^{r}}}
$$

where $p^{r}$ labels the $r$ th repeat of prime cycle $p$.

\section{Trace evaluated at a fixed point to all orders}

We now derive the perturbative expansion for a fixed point $(n=1)$ to all orders in $\sigma$. A fixed point and its repeats are of particular interest having the same interaction at every site, as does the usual field theory. What we do here is to formulate (and partially solve, in the sense of determing a few orders of the exact perturbation theory expansion) the field theory on finite periodic 1-dimensional discrete chains.

Defining $y=f(x)-x$, we can write the fixed point trace as

$$
\operatorname{tr} \mathcal{L}_{\sigma}=\int d x \delta_{\sigma}(f(x)-x)=\int d y \frac{1}{\left|y^{\prime}(x)\right|} \delta_{\sigma}(y) .
$$

Expanding the kernel $\delta_{\sigma}(y)$ as in (3) and integrating by parts, we see that all is well if we know the $d / d x$ derivatives of $1 / y^{\prime}(x)$. Replacing

$$
\frac{d}{d y} \rightarrow \frac{\partial x}{\partial y} \frac{d}{d x}=\frac{1}{y^{\prime}(x)} \frac{d}{d x}
$$

we obtain in the saddlepoint approximation contributions to each fixed point of $f$ evaluated recursively as derivatives of $1 / y^{\prime}(x)$

$$
\int d x \delta^{(k)}(y)=\sum_{x: y(x)=0}(-1)^{k} \frac{d^{k}}{d y^{k}} \frac{1}{\left|y^{\prime}(x)\right|}=\sum_{x: y(x)=0}\left(-\frac{1}{y^{\prime}(x)} \frac{d}{d x}\right)^{k} \frac{1}{\left|y^{\prime}(x)\right|} .
$$


The $d / d y$ derivatives of $1 / y^{\prime}$ are related to the $d / d x$ derivatives of the map $f$ by

$$
\begin{aligned}
\frac{1}{y^{\prime}} & =\frac{1}{f^{\prime}(x)-1} \\
\frac{d}{d y} \frac{1}{y^{\prime}} & =-\frac{f^{\prime \prime}}{\left(y^{\prime}\right)^{3}} \\
\frac{d^{2}}{d y^{2}} \frac{1}{y^{\prime}} & =-\frac{f^{\prime \prime \prime}}{\left(y^{\prime}\right)^{4}}+3 \frac{\left(f^{\prime \prime}\right)^{2}}{\left(y^{\prime}\right)^{5}} \\
\frac{d^{3}}{d y^{3}} \frac{1}{y^{\prime}} & =-\frac{f^{\prime \prime \prime \prime}}{\left(y^{\prime}\right)^{5}}+\frac{10 f^{\prime \prime} f^{\prime \prime \prime}}{\left(y^{\prime}\right)^{6}}-\frac{15\left(f^{\prime \prime}\right)^{3}}{\left(y^{\prime}\right)^{7}}
\end{aligned}
$$

Here, and also later, we have relegated the fourth and fifth derivatives (A.1) to the Appendix. For example, for the second derivative of the delta function we have

$$
\int d x \delta^{(2)}(y)=\sum_{x: y(x)=0} \frac{1}{\left|y^{\prime}\right|}\left(3 \frac{\left(y^{\prime \prime}\right)^{2}}{\left(y^{\prime}\right)^{4}}-\frac{y^{\prime \prime \prime}}{\left(y^{\prime}\right)^{3}}\right),
$$

where the sum is over all fixed points $f(x)=x$. In general, for $n \geq 1$

$$
(-1)^{n} \frac{d^{n}}{d y^{n}} \frac{1}{\left|y^{\prime}\right|}=\frac{1}{\left|y^{\prime}\right|} \sum_{\left\{k_{\ell}\right\}} \frac{\left(2 n-k_{1}\right) !}{\left(-y^{\prime}\right)^{2 n-k_{1}}} \prod_{\ell \geq 2} \frac{f^{(\ell)^{k_{\ell}}}}{(\ell !)^{k_{\ell}} k_{\ell} !} .
$$

where $f^{(\ell)}$ is the $\ell$-th derivative and the sum is over all sets $\left\{k_{\ell}\right\}$ satisfying $\ell \geq 1$, $k_{\ell} \geq 0, \sum k_{\ell}=n$, and $\sum \ell k_{\ell}=2 n$, that is, all partitions of $2 n$ into $n$ terms. The product contains only the finitely many nonzero $k_{\ell}$ for $\ell \geq 2$. This formula may be proved in a staightforward manner by induction. It may be simplified substantially by defining a generating function

$$
\begin{aligned}
\mathcal{F}(z, x) & =\left.\sum_{n=1}^{\infty} z^{n} \frac{d^{n-1}}{d y^{n-1}} \frac{1}{y^{\prime}(x)}\right|_{x: y(x)=0} \\
& =\exp \left(-z^{-1} \sum_{j=2}^{\infty} \frac{f^{(n)} z^{n} \partial_{y^{\prime}}^{n}}{n !}\right) \frac{z}{y^{\prime}},
\end{aligned}
$$

where $\partial_{y^{\prime}}=\partial / \partial y^{\prime}$ acts only on $y^{\prime}$. The sum in the exponential is formally a Taylor series expansion of

$$
\mathcal{F}(z, x)=\exp \left\{-z^{-1}\left[f\left(x-\partial_{u}\right)+\Lambda \partial_{u}-x\right]\right\} \frac{1}{u},
$$

with $u=y^{\prime} / z, f^{\prime}(x)=\Lambda$ and $f(x)=x$.

\section{Smooth conjugacies}

The next step injects into field theory a method standard in the analysis of fixed points and construction of normal forms for bifurcations, see refs. [9]- 18$]$. The idea is to perform a smooth nonlinear change of coordinates that flattens out the vicinity of a fixed point and makes the map linear in an open neighborhood. This can be implemented only for an isolated nondegenerate fixed point (otherwise higher terms will contribute to the normal form expansion around the point), and only in a finite neigborhood of a point, as the conjugating function in general has a finite radius of convergence. Later we extend the method to periodic orbits, which are fixed points of the $n$th iterated map. 


\subsection{Fixed points}

Let the fixed point of $f(x)$ be $x=0$ and the stability of that point be $\Lambda=f^{\prime}(0)$. If $|\Lambda| \neq 1$, there exists a smooth conjugation $h(x)$ satisfying $h(0)=0$ such that:

$$
f(x)=h\left(\Lambda h^{-1}(x)\right) .
$$

In several dimensions, $\Lambda$ is replaced by the Jacobian matrix, and one has to check that its eigenvalues are non-resonant, that is, there is no integer linear relation between their logarithms. If $h(x)$ is a conjugation, so is any scaling $h(\alpha x)$ of the function for a real number $\alpha$. Hence the value of $h^{\prime}(0)$ is not determined by the functional equation; we shall set $h^{\prime}(0)=1$.

To compute the conjugation $h$ we use the functional equation $h(\Lambda x)=f(h(x))$ and the expansions

$$
\begin{aligned}
& f(x)=\Lambda x+x^{2} f_{2}+x^{3} f_{3}+\ldots \\
& h(x)=x+x^{2} h_{2}+x^{3} h_{3}+\ldots .
\end{aligned}
$$

In the present context absorbing the factorials into the definition of expansion coefficients turns out to be more convenient than the standard Taylor expansion. Equating recursively coefficients in expansions

$$
\begin{aligned}
h(\Lambda u)-\Lambda h(u) & =\sum_{n=2}^{\infty} f_{m}(h(u))^{m} \\
\sum_{n=2}^{\infty}\left(\Lambda^{n}-\Lambda\right) h_{n} u^{n} & =\sum_{m=2}^{\infty} f_{m} u^{m}\left(1+\sum_{k=2}^{\infty} h_{k} u^{k-1}\right)^{m}
\end{aligned}
$$

yields

$$
h_{2}=\frac{f_{2}}{\Lambda(\Lambda-1)}, \quad h_{3}=\frac{2 f_{2}^{2}+\Lambda(\Lambda-1) f_{3}}{\Lambda^{2}(\Lambda-1)\left(\Lambda^{2}-1\right)}, \quad \ldots
$$

Noting that the left-hand-side of (18) generates denominators in (19) are the same as those appearing in the Euler formula

$$
\begin{aligned}
\prod_{k=0}^{\infty}\left(1+t u^{k}\right) & =1+\frac{t}{1-u}+\frac{t^{2} u}{(1-u)\left(1-u^{2}\right)}+\frac{t^{3} u^{3}}{(1-u)\left(1-u^{2}\right)\left(1-u^{3}\right)} \cdots \\
& =\sum_{k=0}^{\infty} t^{k} \frac{u^{\frac{k(k-1)}{2}}}{(1-u) \cdots\left(1-u^{k}\right)}, \quad|u|<1
\end{aligned}
$$

we find it convenient to factorize $h_{n}$ as

$$
h_{n}=\frac{b_{n}}{D_{n}}, \quad D_{n}=\left(1-\frac{1}{\Lambda}\right)\left(1-\frac{1}{\Lambda^{2}}\right) \cdots\left(1-\frac{1}{\Lambda^{n-1}}\right) \Lambda^{\frac{(n+2)(n-1)}{2}} .
$$

Computer algebra then yields

$$
\begin{aligned}
& b_{2}=f_{2} \\
& b_{3}=2 f_{2}^{2}+\Lambda(\Lambda-1) f_{3} \\
& b_{4}=(5+\Lambda) f_{2}^{3}-\Lambda\left(5-2 \Lambda-3 \Lambda^{2}\right) f_{2} f_{3}+\Lambda^{2}(\Lambda-1)\left(\Lambda^{2}-1\right) f_{4} .
\end{aligned}
$$

The expressions A.2 for $b_{5}$ and $b_{6}$ are given in the Appendix. 


\subsection{Longer cycles}

Now that we have constructed the conjugation function for a fixed point, we turn to the problem of constructing it for periodic orbits. Each point around the cycle has a differently distorted neighborhood, with differing second and higher derivatives, so we need to compute a conjugation function $h_{a}$ at each cycle point $x_{a}$. We expand the map $f$ around each cycle point along the cycle,

$$
y_{a}(\phi)=f_{a}(\phi)-x_{a+1}=\phi f_{a, 1}+\phi^{2} f_{a, 2}+\ldots
$$

where $x_{a}$ is a point on the cycle, $f_{a}(\phi)=f\left(x_{a}+\phi\right)$ is centered on the deterministic orbit, and the index $k$ in $f_{a, k}$ refers to the $k$ th order in the (modified) Taylor expansion (17).

For a periodic orbit the conjugation formula (16) generalizes to

$$
f_{a}(\phi)=h_{a+1}\left(f_{a}^{\prime}(0) h_{a}^{-1}(\phi)\right)
$$

at each point. The conjugationg functions $h_{a}$ are obtained in the same way as before, by equating coefficients of Taylor series, and assuming that the cycle stability is not marginal, $|\Lambda| \neq 1$. The explicit expressions for $h_{a}$ in terms of $f$ are obtained by iterating around the whole cycle,

$$
f^{n}\left(x_{a}+\phi\right)=h_{a}\left(\Lambda h_{a}^{-1}(\phi)\right)+x_{a},
$$

so each $h_{a}$ function is given by the derivatives given in the previous section acting on $f^{n}$, evaluated at each cycle point a (recursive formulas for evaluation of such derivatives are given in Appendix A of ref. [1]). Again we have the freedom to set $h_{a}^{\prime}(0)=1$ for all $a$.

We shall also find it convenient to define partial stabilities along cycle laps

$$
\begin{aligned}
& f^{j}\left(x_{a}+\phi\right)=x_{b}+h_{b}\left(\Lambda_{j} h_{a}^{-1}(\phi)\right) \\
& f^{k}\left(x_{b}+\phi\right)=x_{a}+h_{a}\left(\Lambda_{k} h_{b}^{-1}(\phi)\right)
\end{aligned}
$$

where

$$
\Lambda_{j}=\prod_{c=a}^{b-1} f^{\prime}\left(x_{c}\right), \quad \Lambda_{k}=\prod_{c=b}^{a-1} f^{\prime}\left(x_{c}\right)
$$

and $\Lambda_{j} \Lambda_{k}=\Lambda$.

At this point we note that while in the deterministic case the cycle weight is given by the cycle stability, a quantity invariant under all smooth coordinate transfomations, the higher order corrections are not invariant. The noise is defined with respect to a particular coordinate system, and it has no invariant meaning.

\section{Trace formula for repeats of periodic orbits}

What is gained by rewriting the perturbation expansion for $f$ in terms of the equally messy perturbation expansion for the conjugacy function $h$ ? Once the neighborhood of a fixed point is linearized, the repeats of it are trivialized; from the conjugation formula (16) one can compute the derivatives of a function composed with itself $r$ times:

$$
f^{r}(x)=h\left(\Lambda^{r} h^{-1}(x)\right) .
$$

One can already discern the form of the expansion for arbitrary repeats; the answer will depend on the conjugacy function $h(x)$ computed for a single repeat, and all the dependence on the repeat number will be carried by factors polynomial in $\Lambda^{r}$, a result that emerged as a surprise in the Feynman diagrammatic approach [1]. 


\subsection{Repeats of fixed points}

The above observation enables us to move from a field theory constructed at a single point to a field theory which is translationally invariant on a periodic chain of arbitrary length $r$. The first and the second derivatives are

$$
\begin{aligned}
\frac{d}{d x} f^{r}(x) & =h^{\prime}\left(\Lambda^{r} h^{-1}(x)\right) \Lambda^{r} \frac{1}{h^{\prime}\left(h^{-1}(x)\right)} \\
\frac{d^{2}}{d x^{2}} f^{r}(x) & =\frac{\Lambda^{2 r} h^{\prime \prime}\left(\Lambda^{r} h^{-1}(x)\right)}{h^{\prime}\left(h^{-1}(x)\right)^{2}}-\frac{\Lambda^{r} h^{\prime \prime}\left(h^{-1}(x)\right) h^{\prime}\left(\Lambda^{r} h^{-1}(x)\right)}{h^{\prime}\left(h^{-1}(x)\right)^{3}} .
\end{aligned}
$$

The third derivative is too long to write down here. Evaluation at the fixed point $x=0$ yields

$$
\begin{aligned}
& \frac{d}{d x} f^{r}(0)=\Lambda^{r}, \quad \frac{d^{2}}{d x^{2}} f^{r}(0)=\Lambda^{r}\left(\Lambda^{r}-1\right) h^{(2)} \\
& \frac{d^{3}}{d x^{3}} f^{r}(0)=\Lambda^{r}\left(\Lambda^{2 r}-1\right) h^{(3)}-3 \Lambda^{r}\left(\Lambda^{r}-1\right) h^{(2)^{2}} \cdots .
\end{aligned}
$$

(For brevity we shall often replace $h^{(r)}(0) \rightarrow h^{(r)}=r ! h_{r}, f^{(r)}(0) \rightarrow f^{(r)}=r ! f_{r}$ throughout, where the superscript ${ }^{(r)}$ labels the $r$ th coefficient in the standard Taylor expansion.) In general, the $k$ th derivative of a function composed with itself $r$ times is a polynomial in $\Lambda^{r}$ of degree $k$

$$
\frac{d^{k}}{d x^{k}} f^{r}(0)=\sum_{j=1}^{k} c_{j} \Lambda^{j r} .
$$

The coefficients $c_{j}$ are in turn expressed in terms of the first $k$ derivatives $h^{(j)}$ of the conjugation function.

Now we can reevaluate the fixed-point derivatives of sect. 囵 for the case of $r$ th repeat of a fixed point

$$
y(x)=f^{n_{p} r}(x)-x
$$

in terms of the conjugating function $h$ by substituting (23) into (11):

$$
\begin{gathered}
\left.\frac{1}{3 !} \frac{\partial^{2}}{\partial y^{2}} \frac{1}{y^{\prime}(x)}\right|_{x=0}=\frac{\Lambda^{r}\left(1+\Lambda^{r}\right)}{\left(\Lambda^{r}-1\right)^{3}}\left(2 h_{2}^{2}-h_{3}\right) \\
\left.\frac{1}{4 !} \frac{\partial^{3}}{\partial y^{3}} \frac{1}{y^{\prime}(x)}\right|_{x=0}=-5 \Lambda^{r} \frac{\left(\Lambda^{r}+1\right)^{2}}{\left(\Lambda^{r}-1\right)^{4}} h_{2}^{3}+\Lambda^{r} \frac{5 \Lambda^{2 r}+8 \Lambda^{r}+5}{\left(\Lambda^{r}-1\right)^{4}} h_{2} h_{3} \\
-\Lambda^{r} \frac{\Lambda^{2 r}+\Lambda^{r}+1}{\left(\Lambda^{r}-1\right)^{4}} h_{4}
\end{gathered}
$$

The 4th and 5th order derivatives (A.3) are relegated to the Appendix.

\subsection{Repeats of periodic orbits}

The above fixed-point formulas carry over to the case of general periodic orbits, if $f$ is identified with the $n$th iterated map at a particular point along the cycle $a$ from which we define $h_{a}$ as in (21). The second derivative required for the $\sigma^{2}$ correction follows immediately, with (25) evaluated for each cycle point in the prime cycle trace (6). It is easily checked that this second derivative (25) has exactly the same form as what we obtained in a much more involved manner in ref. [1]. The somewhat more complicated sum of Feynman diagrams is here replaced by (25), the conjugation 
function determined from the iterated map, with $h$ carrying all dependence on the higher derivatives of the original map.

The factorisation is not quite as simple for higher orders in the trace formula. For a general non-Gaussian case at order $\sigma^{3}$ we have three terms from (26), each of which may be resummed separately. The calculation is entirely analogous to the second order calculation in ref. [1]. Powers of $\Lambda^{r}$ are moved around in order to express (26) in terms of $|\Lambda|^{-r}<1$ and generate convergent geometric series; each of the three terms is expanded using identities such as

$$
3 \frac{(1+x)^{2}}{(1-x)^{4}}=\sum_{k=0}^{\infty} k\left(1+2 k^{2}\right) x^{k}
$$

so that repeats $r$ can be resummed. (The remaining identities (A.6)-(A.8) used here

are relegated to the Appendix.) This yields the saddlepoint approximation to the spectral determinant in product form up to order $\sigma^{3}$

$$
\begin{aligned}
\operatorname{det}\left(1-z \mathcal{L}_{\sigma}\right)=\prod_{p} \prod_{k=0}^{\infty}\left(1-t_{p, k}\right) \\
t_{p, k}=\frac{z^{n_{p}}}{\left|\Lambda_{p}\right|} \frac{1}{\Lambda_{p}^{k}} \exp \left(\frac{1}{2} a_{2} \sigma^{2} w_{p, k}^{(2)}+\frac{1}{3 !} a_{3} \sigma^{3} w_{p, k}^{(3)}+O\left(\sigma^{4}\right)\right) \\
w_{p, k}^{(2)}=(k+1)^{2} \sum_{a}\left(2 h_{a}^{(2)^{2}}-h_{a}^{(3)}\right) \\
w_{p, k}^{(3)}=-\frac{k\left(1+2 k^{2}\right)}{3} \sum_{a} h_{a}^{(2)^{3}}+(1+k)\left(5+6 k+3 k^{2}\right) \sum_{a} h_{a}^{(2)} h_{a}^{(3)} \\
\quad-\frac{(1+k)\left(2+2 k+k^{2}\right)}{2} \sum_{a} h_{a}^{(4)}
\end{aligned}
$$

The meaning of this factorization is that in the saddlepoint approximation the spectral determinant is composed of local spectra evaluated for each prime cycle separately, with the index $k$ labelling the $k$ th local eigenvalue.

From $\sigma^{4}$ onward further terms come into play, as we now describe.

\section{Two-point integrals}

In general, a saddlepoint at each cycle point may be expanded in second and higher derivatives (3), with the trace to $n$th order receiving a single $\delta^{(n)}$ integral contribution as in (6). At fourth order we need to also include the two-point integral contribution (7). Setting $y_{j}=f^{j}\left(x_{a}\right)-x_{b}, y_{k}=f^{k}\left(x_{b}\right)-x_{a}$, and integrating by parts, we obtain for each pair of points along the cycle

$$
\int \frac{d y_{j} d y_{k}}{\left|f^{j^{\prime}}\left(x_{a}\right) f^{k^{\prime}}\left(x_{b}\right)-1\right|} \delta^{(2)}\left(y_{j}\right) \delta^{(2)}\left(y_{k}\right)=\frac{\partial^{2}}{\partial y_{j}^{2}} \frac{\partial^{2}}{\partial y_{k}^{2}} \frac{1}{\left|f^{j^{\prime}}\left(x_{a}\right) f^{k^{\prime}}\left(x_{b}\right)-1\right|} .
$$

This expression looks innocent enough, but its evaluation requires attention to some subtle points, and more algebra than a human would be willing to handle. Performing the required derivatives taxes the ability of general purpose symbolic algebra packages, so the results in this section are obtained with a program written in C. 
Consider evaluating partial derivatives of a function $q$ with respect to, say $y_{j}$ keeping $y_{k}$ constant in terms of $x_{a}$ and $x_{b}$ derivatives. The solution is to consider an arbitrary infinitesimal transformation which respects the $d y_{k}=0$ constraint:

$$
\begin{aligned}
d q & =\frac{\partial q}{\partial x_{a}} d x_{a}+\frac{\partial q}{\partial x_{b}} d x_{b} \\
d y_{j} & =\frac{\partial y_{j}}{\partial x_{a}} d x_{a}+\frac{\partial y_{j}}{\partial x_{b}} d x_{b} \\
d y_{k} & =\frac{\partial y_{k}}{\partial x_{a}} d x_{a}+\frac{\partial y_{k}}{\partial x_{b}} d x_{b}=0
\end{aligned}
$$

These equations can then be solved for the ratio of $d q$ and $d y_{j}$,

$$
\frac{\partial q}{\partial y_{j}}=\frac{\frac{\partial q}{\partial x_{a}} \frac{\partial y_{k}}{\partial x_{b}}-\frac{\partial q}{\partial x_{b}} \frac{\partial y_{k}}{\partial x_{a}}}{\frac{\partial y_{j}}{\partial x_{a}} \frac{\partial y_{k}}{\partial x_{b}}-\frac{\partial y_{j}}{\partial x_{b}} \frac{\partial y_{k}}{\partial x_{a}}}
$$

and similarly for $\partial q / \partial y_{k}$.

Sixth order corrections require a three point integral, which we leave as an exercise for the reader.

If for some reason the noise kernel contains a linear $a_{1} \sigma$ term in the expansion (3), for example if the evolution operator is weighted, and the shift that this causes to the saddlepoint is not taken into account, the relevant second order correction includes the term

$$
\begin{aligned}
& \frac{\partial}{\partial y_{j}} \frac{\partial}{\partial y_{k}} \frac{1}{\left(\partial f^{j}\left(x_{a}\right) / \partial x_{a}\right)\left(\partial f^{k}\left(x_{b}\right) / \partial x_{b}\right)-1 \mid} \\
= & \frac{\Lambda}{(\Lambda-1)^{3}}\left[(\Lambda+1) h_{a}^{(2)} h_{b}^{(2)}+2 \Lambda_{k} h_{a}^{(2)^{2}}+2 \Lambda_{j} h_{b}^{(2)^{2}}-\Lambda_{k} h_{a}^{(3)}-\Lambda_{j} h_{b}^{(3)}\right]
\end{aligned}
$$

This we have written down just to illustrate the form of a 2-point term. In our application the saddlepoint condition sets $a_{1}=0$, and what is really required is the two-point fourth order correction (A.4) and (in case of non-Gaussian noise) the twopoint fifth order correction (A.5). The corresponding expressions are too cumbersome for the main text, so we relegate them to Appendix.

There are some convenient cross-checks on the algebra. The results reduce to those of (26) and (A.3) for single repeat $r=1$, by setting $h_{a}=h_{b}=h, \Lambda_{j}=\Lambda$, $\Lambda_{k}=1$, and remebering that in order to retain integer coefficients, we have here replaced power series coefficients $h_{r}$ by the Taylor expansion derivatives $h^{(r)}=r ! h_{r}$. This reduction to the one-point case also means that for the Gaussian case $\left(a_{2}=1\right.$, $a_{4}=3$ ), expression (7) can be written as an unrestricted double sum,

$$
\frac{\sigma^{4}}{8} \sum_{x \in \text { Fix } f^{n}} \sum_{a b} \frac{\partial^{2}}{\partial y_{j}^{2}} \frac{\partial^{2}}{\partial y_{k}^{2}} \frac{1}{\left|\left(\partial f^{j}\left(x_{a}\right) / \partial x_{a}\right)\left(\partial f^{k}\left(x_{b}\right) / \partial x_{b}\right)-1\right|}
$$

where it is understood that $j=n$ and $k=0$ when $a=b$.

Now the stage is set to return to the investigations of ref. [1], where the $\sigma^{2}$ term was evaluated anlytically, while the $\sigma^{4}$ term was only estimated from the numerically computed leading eigenvalue.

\section{Numerical tests}

Here we continue the calculations of Sect. 5 of ref. [1], where more details and discussion may be found. We test our perturbative expansion on the repeller of the 1dimensional map $f(x)=20\left[(1 / 2)^{4}-((1 / 2)-x)^{4}\right]$. This repeller is a nice example of an 
"Axiom A" expanding system of bounded nonlinearity and complete binary symbolic dynamics, for which the deterministic evolution operator eigenvalues converge superexponentially with the cycle length. We compute the leading eigenvalue of the evolution operator (the repeller escape rate) in the presence of Gaussian noise, using two complementary approaches. The perturbative result in terms of periodic orbits and the weak noise corrections is compared to a numerical eigenvalue obtained in ref. [1] by approximating the dynamics by a finite matrix. In the preceding paper we compared the numerical eigenvalue with the $\sigma^{2}$ result and estimated the coefficient of $\sigma^{4}$ to be approximately 38 . Here we compute the order $\sigma^{4}$ coefficient to 14 digits accuracy, and estimate the $\sigma^{6}$ term. In the Feynman diagram language, the $\sigma^{4}$ contribution is a "2-loop" calculation, albeit one of relatively simple kind where space integrals are replaced by discrete sums over a finite periodic chains. The conjugation functions (21) at each point around the cycle are obtained by a recursive evaluation of (18), and then substituted into (25) for the second order and (A.5) for the fourth order. The expansion for the spectral determinant is obtained by differentiating $\ln \operatorname{det}\left(1-z \mathcal{L}_{\sigma}\right)=\operatorname{tr} \ln \left(1-z \mathcal{L}_{\sigma}\right)$ with respect to $z$, multiplying through by the determinant, and equating coefficients order by order in $z$ and $\sigma$, as in ref. [1]. That is, we define coefficients

$$
\operatorname{tr} \mathcal{L}_{\sigma}^{n}=\sum_{j=0}^{\infty} C_{n, j} \sigma^{j}, \quad \operatorname{det}\left(1-z \mathcal{L}_{\sigma}\right)=1-\sum_{n=1}^{\infty} \sum_{j=0}^{\infty} Q_{n, j} z^{n} \sigma^{j},
$$

and obtain the cumulants $Q_{n, j}$ recursively as

$$
\begin{aligned}
Q_{n, 0} & =\frac{1}{n}\left[C_{n, 0}-\sum_{k=1}^{n-1} Q_{k, 0} C_{n-k, 0}\right] \\
Q_{n, 2} & =\frac{1}{n}\left[C_{n, 2}-\sum_{k=1}^{n-1}\left(Q_{k, 2} C_{n-k, 0}+Q_{k, 0} C_{n-k, 2}\right)\right] \\
Q_{n, 4} & =\frac{1}{n}\left[C_{n, 4}-\sum_{k=1}^{n-1}\left(Q_{k, 4} C_{n-k, 0}+Q_{k, 2} C_{n-k, 2}+Q_{k, 0} C_{n-k, 4}\right)\right],
\end{aligned}
$$

where it is understood that the sums do not contribute when $n=1$.

The noiseless, zeroth order eigenvalue equation $\operatorname{det}\left(1-z \mathcal{L}_{0}\right)=0$ is solved by Newton's method to find the leading eigenvalue $\nu_{0}=z^{-1}$ at $\sigma=0$, and the higher order equations give the noise corrections $\nu(\sigma)=\nu_{0}+\nu_{2} \sigma^{2}+\nu_{4} \sigma^{4}+O\left(\sigma^{6}\right)$ in terms of $\nu_{0}$ and the expansion of the determinant. Expanding the spectral determinant order by order in $z$ and $\sigma$ we find

$$
\operatorname{det}\left(1-z \mathcal{L}_{\sigma}\right)=F-F_{10} z-F_{02} \sigma^{2}-F_{20} z^{2}-F_{12} z \sigma^{2}-F_{04} \sigma^{4}-\ldots
$$

where the expansion coefficients are

$$
\begin{aligned}
& F=1-\sum_{m=1}^{n} \frac{Q_{m, 0}}{\nu_{0}^{m}}, \quad F_{10}=\sum_{m=1}^{n} \frac{m Q_{m, 0}}{\nu_{0}^{m-1}}, \quad F_{02}=\sum_{m=1}^{n} \frac{Q_{m, 2}}{\nu_{0}^{m}} \\
& F_{20}=\sum_{m=2}^{n} \frac{m(m-1) Q_{m, 0}}{2 \nu_{0}^{m-2}}, \quad F_{12}=\sum_{m=1}^{n} \frac{m Q_{m, 2}}{2 \nu_{0}^{m-1}}, \quad F_{04}=\sum_{m=1}^{n} \frac{Q_{m, 4}}{\nu_{0}^{m}}
\end{aligned}
$$

are obtained from derivatives of (8). Finally we expand $\nu=z^{-1}$ in powers of $\sigma^{2}$ and equate coefficients of powers of $\sigma$ to obtain

$$
\nu_{2}=\frac{F_{02} \nu_{0}^{2}}{F_{10}}, \quad \nu_{4}=\frac{F_{20} F_{02}^{2}-2 F_{12} F_{10} F_{02}+F_{04} F_{10}^{2}+F_{10} F_{02}^{2} \nu_{0}}{F_{10}^{3}} \nu_{0}^{2}
$$




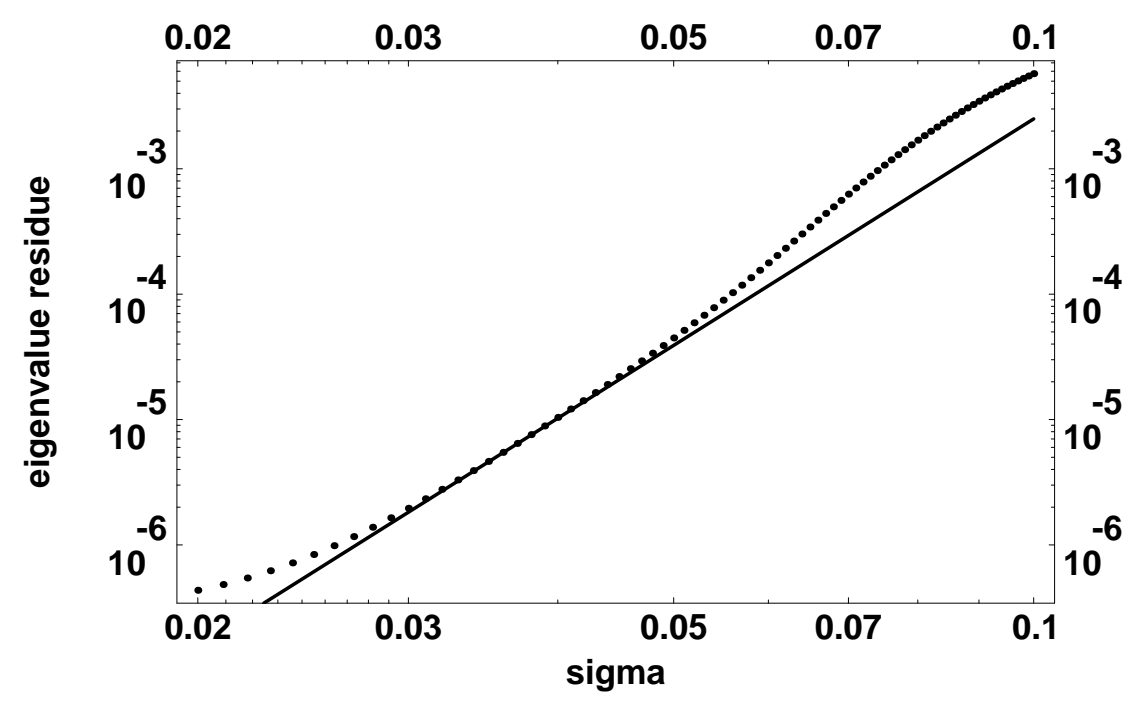

Figure 1. Numerically computed eigenvalue minus known terms, that is, $\nu(\sigma)-\nu_{0}-\sigma^{2} \nu_{2}-\sigma^{4} \nu_{4}$ (dots), together with estimated next term $2700 \sigma^{6}$ (solid line). Deviations occur at small $\sigma$ due to discretization errors in the numerical eigenvalue of a few times $10^{-7}$, and at large $\sigma$ due to further omitted terms $\left(\sigma^{8} \ldots\right)$.

\begin{tabular}{llll}
\hline$n$ & $\nu_{0}$ & $\nu_{2}$ & $\nu_{4}$ \\
\hline 1 & 0.308 & 0.42 & 2.2 \\
2 & 0.37140 & 1.422 & 32.97 \\
3 & 0.3711096 & 1.43555 & 36.326 \\
4 & 0.371110995255 & 1.435811262 & 36.3583777 \\
5 & 0.371110995234863 & 1.43581124819737 & 36.35837123374 \\
6 & 0.371110995234863 & 1.43581124819749 & 36.358371233836 \\
\hline
\end{tabular}

Table 1. Significant digits of the leading deterministic eigenvalue and its $\sigma^{2}$ and $\sigma^{4}$ coefficients, calculated from the spectral determinant as a function of the cycle truncation length $n$. Note the super-exponential convergence of all coefficients (the $n=6$ result is here limited by the machine precision).

The perturbative corrections to the leading eigenvalue (escape rate) of the weaknoise evolution operator are given in table 1, showing super-exponential convergence with the truncation cycle length $n$. The super-exponential convergence has been proven for the deterministic, $\nu_{0}$ part of the eigenvalue [19, 20], but has not been studied for noisy kernels. It is seen that a good first approximation is obtained already at $n=2$, using only 3 prime cycles, and $n=6$ (23 prime cycles in all) is in this example sufficient to exhaust the limits of double precision arithmetic. The exact value of $\nu_{4}=36.358 \ldots$ is encouragingly close to our previous numerical estimate [1] of 38. As in the preceding paper [1], we subtract the known terms in the expansion from the numerically evaluated eigenvalue, and obtain a good fit to the next term, approximately $2700 \sigma^{6}$, see fig. 1. 1 . 


\section{Summary and outlook}

We have formulated a perturbation theory of stochastic trace formulas based on smooth nonlinear field transformations around infinitely many chaotic saddle points (unstable periodic orbits). In contrast to previous perturbative expansions around vacua and instanton solutions, the location and local properties of each saddlepoint must be found numerically. In addition, every interaction term depends on the position, hence also the classical periodic solution at which it is evaluated.

Even though in the model calculation we have chosen a Gaussian one-step noise, the accumulated noise along a trajectory is distorted by the nonlinear flow, and orbitby-orbit the noise corrections are not Gaussian. Gaussian noise has thus no privilaged role in nonlinear dynamical systems.

The key idea is this: Instead of separating the action into a quadratic and "interaction" parts, we first perform a nonlinear field transformation ("smooth conjugation") which turns the saddle point into an exact quadratic form. The price one pays for this is the Jacobian of the nonlinear field transformation - but it turns out that the perturbation expansion of this Jacobian in terms of the conjugating function is order-by-order considerably more compact than the Feyman-diagrammatic expansion.

We have resummed repeats of prime cycles to third order in the noise strength, carried out the numerical tests to fourth order, given the trace formula for general periodic orbits to fifth order, and for a fixed point to all orders.

The rapid rate of increase of the numerical coefficients confirms the expectation that the series is asymptotic, and is to be used with caution, as long as no sophisticated summation beyond all orders is implemented.

The smooth conjugacy method of perturbation expansions can be extended to the case of higher dimensions and continuous time dynamics (stochastic flows), but the main interest comes from the observation that we have a new method of evaluating perturbative corrections to saddlepoints of path integrals. In quantum mechanics and field theory the perturbative corrections do matter, and the method might have applications there, in particular to the $\hbar$ expansion of semiclassical periodic orbit theory. If efficient methods are found for computing numerical periodic solutions of spatially extended systems, the method might apply to the field theory as well.

\section{Appendix A. Appendix: Some algebra}

We collect here some of the formulas used in our calculations, but too long for the patience of a casual reader.

Evaluation of the trace of a fixed point (11) continued to 4th and 5th order:

$$
\begin{aligned}
\frac{d^{4}}{d y^{4}} \frac{1}{y^{\prime}}= & -\frac{f^{(5)}}{\left(y^{\prime}\right)^{6}}+\frac{15 f^{\prime \prime} f^{\prime \prime \prime \prime}}{\left(y^{\prime}\right)^{7}}+\frac{10\left(f^{\prime \prime \prime}\right)^{2}}{\left(y^{\prime}\right)^{7}}-\frac{105\left(f^{\prime \prime}\right)^{2} f^{\prime \prime \prime}}{\left(y^{\prime}\right)^{8}}+\frac{105\left(f^{\prime \prime}\right)^{4}}{\left(y^{\prime}\right)^{9}} \\
\frac{d^{5}}{d y^{5}} \frac{1}{y^{\prime}}= & -\frac{f^{(6)}}{\left(y^{\prime}\right)^{7}}+\frac{21 f^{\prime \prime} f^{(5)}}{\left(y^{\prime}\right)^{8}}+\frac{35 f^{\prime \prime \prime} f^{\prime \prime \prime \prime}}{\left(y^{\prime}\right)^{8}}-\frac{210\left(f^{\prime \prime}\right)^{2} f^{\prime \prime \prime \prime}}{\left(y^{\prime}\right)^{9}} \\
& -\frac{280 f^{\prime \prime}\left(f^{\prime \prime \prime}\right)^{2}}{\left(y^{\prime}\right)^{9}}+\frac{1260\left(f^{\prime \prime}\right)^{3} f^{\prime \prime \prime}}{\left(y^{\prime}\right)^{10}}-\frac{945\left(f^{\prime \prime}\right)^{5}}{\left(y^{\prime}\right)^{11}} .
\end{aligned}
$$

Perturbative formulas for 5 th and 6th order conjugacy coefficients, continuation of (20):

$$
b_{5}=2\left(7+3 \Lambda+2 \Lambda^{2}\right) f_{2}^{4}+\Lambda\left(-21+\Lambda+6 \Lambda^{2}+11 \Lambda^{3}+3 \Lambda^{4}\right) f_{2}^{2} f_{3}
$$


Stochastic trace formulas

$$
\begin{aligned}
& +2 \Lambda^{2}(\Lambda-1)^{2}\left(3+5 \Lambda+4 \Lambda^{2}+2 \Lambda^{3}\right) f_{2} f_{4}+3 \Lambda^{2}(\Lambda-1)\left(\Lambda^{3}-1\right) f_{3}^{2} \\
& +\Lambda^{3}(\Lambda-1)\left(\Lambda^{2}-1\right)\left(\Lambda^{3}-1\right) f_{5} \\
b_{6}= & 2\left(21+14 \Lambda+14 \Lambda^{2}+8 \Lambda^{3}+3 \Lambda^{4}\right) f_{2}^{5} \\
& +\Lambda\left(-84-21 \Lambda-6 \Lambda^{2}+25 \Lambda^{3}+44 \Lambda^{4}+27 \Lambda^{5}+14 \Lambda^{6}+\Lambda^{7}\right) f_{2}^{3} f_{3} \\
& +2 \Lambda^{2}\left(14+\Lambda-7 \Lambda^{2}-9 \Lambda^{3}-11 \Lambda^{4}+\Lambda^{5}+\Lambda^{6}+7 \Lambda^{7}+3 \Lambda^{8}\right) f_{2}^{2} f_{4} \\
& +(-1+\Lambda)^{2} \Lambda^{2} f_{2}\left[\left(28+43 \Lambda+50 \Lambda^{2}+43 \Lambda^{3}+22 \Lambda^{4}+6 \Lambda^{5}\right) f_{3}^{2}\right. \\
& \left.+\Lambda\left(-7-12 \Lambda-10 \Lambda^{2}-3 \Lambda^{3}+7 \Lambda^{4}+10 \Lambda^{5}+10 \Lambda^{6}+5 \Lambda^{7}\right) f_{5}\right] \\
& +(-1+\Lambda)^{3} \Lambda^{3}\left(1+\Lambda+\Lambda^{2}+\Lambda^{3}\right) \\
& \times\left[\left(7+7 \Lambda+4 \Lambda^{2}\right) f_{3} f_{4}+\Lambda\left(-1-\Lambda+\Lambda^{3}+\Lambda^{4}\right) f_{6}\right] .
\end{aligned}
$$

Continuation of the derivative evaluation 26 - the 4 th and 5 th order derivatives:

$$
\begin{aligned}
\left.\frac{1}{5 !} \frac{\partial^{4}}{\partial y^{4}} \frac{1}{y^{\prime}(x)}\right|_{x=0}= & \frac{\Lambda^{r}\left(1+\Lambda^{r}\right)}{\left(\Lambda^{r}-1\right)^{5}}\left\{14\left(1+\Lambda^{r}\right)^{2} h_{2}^{4}\right. \\
& -3\left(7+10 \Lambda^{r}+7 \Lambda^{2 r}\right) h_{2}^{2} h_{3}+3\left(1+\Lambda^{r}+\Lambda^{2 r}\right) h_{3}^{2} \\
& \left.+2\left(3+2 \Lambda^{r}+3 \Lambda^{2 r}\right) h_{2} h_{4}-\left(1+\Lambda^{2 r}\right) h_{5}\right\} \\
\left.\frac{1}{6 !} \frac{\partial^{5}}{\partial y^{5}} \frac{1}{y^{\prime}(x)}\right|_{x=0}= & -\frac{\Lambda^{r}}{\left(\Lambda^{r}-1\right)^{6}}\left\{42\left(1+\Lambda^{r}\right)^{4} h_{2}^{5}\right. \\
& -28\left(1+\Lambda^{r}\right)^{2}\left(3+4 \Lambda^{r}+3 \Lambda^{2 r}\right) h_{2}^{3} h_{3} \\
& +14\left(1+\Lambda^{r}\right)^{2}\left(2+\Lambda^{r}+2 \Lambda^{2 r}\right) h_{2}^{2} h_{4} \\
& -\left(7+14 \Lambda^{r}+18 \Lambda^{2 r}+14 \Lambda^{3 r}+7 \Lambda^{4 r}\right) h_{3} h_{4} \\
& +7\left(4+11 \Lambda^{r}+15 \Lambda^{2 r}+11 \Lambda^{3 r}+4 \Lambda^{4 r}\right) h_{2} h_{3}^{2} \\
& -\left(7+12 \Lambda^{r}+12 \Lambda^{2 r}+12 \Lambda^{3 r}+7 \Lambda^{4 r}\right) h_{2} h_{5} \\
& \left.+\left(1+\Lambda^{r}+\Lambda^{2 r}+\Lambda^{3 r}+\Lambda^{4 r}\right) h_{6}\right\} .
\end{aligned}
$$

The two-point fourth order correction (continuation of calculations of sect. 7):

$$
\begin{aligned}
& \left.\frac{\partial^{2}}{\partial y_{j}^{2}} \frac{\partial^{2}}{\partial y_{k}^{2}} \frac{1}{\left|\left(\partial f^{j}\left(x_{a}\right) / \partial x_{a}\right)\left(\partial f^{k}\left(x_{b}\right) / \partial x_{b}\right)-1\right|}\right|_{\text {cycle }} \\
= & \frac{\Lambda}{(\Lambda-1)^{5}}\left\{( 1 + \Lambda ) \left[\Lambda_{k}^{2}\left(60 h_{a}^{(2)^{4}}-72 h_{a}^{(2)^{2}} h_{a}^{(3)}+12 h_{a}^{(2)} h_{a}^{(4)}+9 h_{a}^{(3)^{2}}-h_{a}^{(5)}\right)\right.\right. \\
& \left.+\Lambda_{j}^{2}\left(60 h_{b}^{(2)^{4}}-72 h_{b}^{(2)^{2}} h_{b}^{(3)}+12 h_{b}^{(2)} h_{b}^{(4)}+9 h_{b}^{(3)^{2}}-h_{b}^{(5)}\right)\right] \\
+ & \left(3+10 \Lambda+3 \Lambda^{2}\right)\left[\Lambda_{k} h_{b}^{(2)}\left(12 h_{a}^{(2)^{3}}-9 h_{a}^{(2)} h_{a}^{(3)}+h_{a}^{(4)}\right)\right. \\
& \left.+\Lambda_{j} h_{a}^{(2)}\left(12 h_{b}^{(2)^{3}}-9 h_{b}^{(2)} h_{b}^{(3)}+h_{b}^{(4)}\right)\right] \\
+ & \left.(1+\Lambda)\left(1+10 \Lambda+\Lambda^{2}\right)\left(3 h_{a}^{(2)^{2}}-h_{a}^{(3)}\right)\left(3 h_{b}^{(2)^{2}}-h_{b}^{(3)}\right)\right\},
\end{aligned}
$$

and the two-point fifth order correction is:

$$
\begin{aligned}
& \left.\frac{\partial^{3}}{\partial y_{j}^{3}} \frac{\partial^{2}}{\partial y_{k}^{2}} \frac{1}{\left|\left(\partial f^{j}\left(x_{a}\right) / \partial x_{a}\right)\left(\partial f^{k}\left(x_{b}\right) / \partial x_{b}\right)-1\right|}\right|_{\text {cycle }} \\
= & \frac{\Lambda}{(\Lambda-1)^{6}}\left\{( 1 + \Lambda ) \Lambda _ { k } ^ { 3 } \left(-360 h_{a}^{(2)^{5}}+600 h_{a}^{(2)^{3}} h_{a}^{(3)}-180 h_{a}^{(2)} h_{a}^{(3)^{2}}\right.\right.
\end{aligned}
$$




$$
\begin{aligned}
& \left.-120 h_{a}^{(2)^{2}} h_{a}^{(4)}+30 h_{a}^{(3)} h_{a}^{(4)}+15 h_{a}^{(2)} h_{a}^{(5)}-h_{a}^{(6)}\right) \\
+ & 6\left(1+3 \Lambda+\Lambda^{2}\right) \Lambda_{k}^{2} h_{b}^{(2)}\left(-60 h_{a}^{(2)^{4}}+72 h_{a}^{(2)^{2}} h_{a}^{(3)}-9 h_{a}^{(3)^{2}}-12 h_{a}^{(2)} h_{a}^{(4)}+h_{a}^{(5)}\right) \\
+ & {\left[15\left(1+7 \Lambda+7 \Lambda^{2}+\Lambda^{3}\right) h_{b}^{(2)^{2}}-2\left(2+13 \Lambda+13 \Lambda^{2}+2 \Lambda^{3}\right) h_{b}^{(3)}\right] } \\
& \Lambda_{k}\left(-12 h_{a}^{(2)^{3}}+9 h_{a}^{(2)} h_{a}^{(3)}-h_{a}^{(4)}\right) \\
+ & {\left[15\left(1+18 \Lambda+42 \Lambda^{2}+18 \Lambda^{3}+\Lambda^{4}\right) h_{b}^{(2)^{3}}\right.} \\
& -2\left(5+82 \Lambda+186 \Lambda^{2}+82 \Lambda^{3}+5 \Lambda^{4}\right) h_{b}^{(2)} h_{b}^{(3)} \\
& \left.+\left(1+14 \Lambda+30 \Lambda^{2}+14 \Lambda^{3}+\Lambda^{4}\right) h_{b}^{(4)}\right]\left(-3 h_{a}^{(2)^{2}}+h_{a}^{(3)}\right) \\
+3 & {\left[-30\left(3+17 \Lambda+17 \Lambda^{2}+3 \Lambda^{3}\right) h_{b}^{(2)^{4}}+5\left(19+101 \Lambda+101 \Lambda^{2}+19 \Lambda^{3}\right) h_{b}^{(2)^{2}} h_{b}^{(3)}\right.} \\
& -10\left(1+5 \Lambda+5 \Lambda^{2}+\Lambda^{3}\right) h_{b}^{(3)^{2}} \\
& \left.-2\left(7+33 \Lambda+33 \Lambda^{2}+7 \Lambda^{3}\right) h_{b}^{(2)} h_{b}^{(4)}+\left(1+4 \Lambda+4 \Lambda^{2}+\Lambda^{3}\right) h_{b}^{(5)}\right] \Lambda_{j} h_{a}^{(2)} \\
+ & {\left[-630\left(1+2 \Lambda+\Lambda^{2}\right) h_{b}^{(2)^{5}}+30\left(31+58 \Lambda+31 \Lambda^{2}\right) h_{b}^{(2)^{3}} h_{b}^{(3)}\right.} \\
& -60\left(4+7 \Lambda+4 \Lambda^{2}\right) h_{b}^{(2)} h_{b}^{(3)^{2}}-15\left(11+18 \Lambda+11 \Lambda^{2}\right) h_{b}^{(2)^{2}} h_{b}^{(4)} \\
& \left.\left.+2\left(17+26 \Lambda+17 \Lambda^{2}\right) h_{b}^{(3)} h_{b}^{(4)}+6\left(3+4 \Lambda+3 \Lambda^{2}\right) h_{b}^{(2)} h_{b}^{(5)}-\left(1+\Lambda+\Lambda^{2}\right) h_{b}^{(6)}\right] \Lambda_{j}^{2}\right\} .
\end{aligned}
$$

The repetition number dependent prefactors in $(26)$ are turned into power series in $\Lambda^{r k}$ using identities

$$
\begin{aligned}
& \frac{1+x}{(1-x)^{3}}=\sum_{k=0}^{\infty}(k+1)^{2} x^{k} \\
& \frac{5+8 x+5 x^{2}}{(1-x)^{4}}=\sum_{k \geq 0}(1+k)\left(5+6 k+3 k^{2}\right) x^{k} \\
& \frac{1+x+x^{2}}{(1-x)^{4}}=\frac{1}{2} \sum_{k \geq 0}(1+k)\left(2+2 k+k^{2}\right) x^{k}
\end{aligned}
$$

\section{References}

[1] P. Cvitanović, C.P. Dettmann, R. Mainieri and G. Vattay, Trace formulas for stochastic evolution operators: Weak noise perturbation theory, J. Stat. Phys (1998), to appear; chao-dyn/9807034.

[2] H. Poincaré. Les méthodes nouvelles de la méchanique céleste. Guthier-Villars, Paris, 1892.

[3] P. Gaspard. Chaos, Scattering and Statistical Mechanics. Cambridge University Press, Cambridge, 1997.

[4] P. Cvitanović, et al., Classical and Quantum Chaos, http://www.nbi.dk/ChaosBook, Niels Bohr Institute (Copenhagen 1998).

[5] M. Gutzwiller. Chaos in classical and quantum mechanics. Springer-Verlag, New-York, 1990.

[6] P. Gaspard and D. Alonso. Phys. Rev. A, 47:R3468-R3471, 1993.

[7] N.G. Van Kampen. Stochastic Processes in Physics and Chemistry. North Holland, Amsterdam, 1981.

[8] A. Lasota and M. MacKey. Chaos, Fractals, and Noise; Stochastic Aspects of Dynamics. Springer-Verlag, Berlin, 1994. 
[9] A. Katok and B. Hasselblatt. Introduction to the Modern Theory of Dynamical Systems. Cambridge University Press, Cambridge, 1995.

[10] C. L. Siegel. Iteration of analytic functions. Ann. Math., 43:607-612, 1942.

[11] J. Moser. Ann. Scuola Norm. Super. Pisa, 20:265-315, 1966; 20:499-535, 1966.

[12] S. Sternberg. Amer. J. Math., 79:809, 1957; 80:623, 1958; 81:578, 1959.

[13] K.-T. Chen. Amer. J. Math., 85:693-722, 1963.

[14] G.R. Belitskii. Russian Math. Surveys, 31:107-177, 1978.

[15] A.D. Brjuno. Trans. Moscow Math. Soc., 25:131-288, 1971; 26:199-238, 1972.

[16] C. Van den Broeck. Renormalization of first-passage times for random walks on deterministic fractals. Physical Review A, 40:7334, 1989.

[17] H.A. Lauwerier. In V. Holden, editor, Chaos, page 39. Princeton University Press, 1986.

[18] I. Gumowski and C. Mira. Recurrances and Discrete Dynamical Systems. Springer-Verlag, Berlin, 1980.

[19] D. Ruelle, Ergod. The. and Dynam. Sys., 2:99, 1976.

[20] H.H. Rugh, Nonlinearity, 5:1237, 1992. 